\title{
Der Gordische Knoten
}

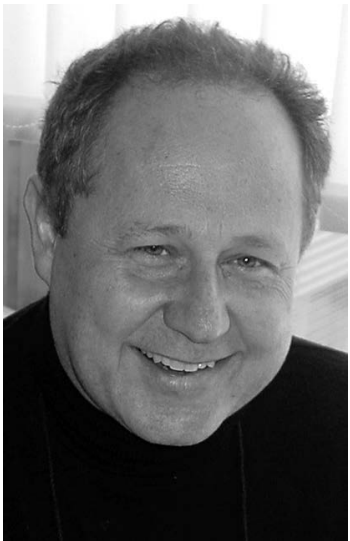

Prof. Dr. med. Manfred Wildner
Der sprichwörtliche Gordische Knoten hat seinen historisch-mythologischen Bezug zu König Gordios von Phrygien in der heutigen Zentraltürkei. Die Deichsel seines Wagens war mit kunstvoll verschlungenen Seilen mit dem Zugjoch für die Pferde verbunden. Nach seiner Prophezeiung würde derjenige, der diesen Knoten zu lösen vermochte, die Herrschaft über Kleinasien erringen. Alexander der Große löste im Jahr 333 v. Chr. diese Aufgabe auf seine Weise: Er durchschlug den Knoten mit dem Schwert und eroberte Kleinasien, das persische Großreich und gelangte bis an den Indus. Zumindest ist das die Variante des Plutarch. Arrian beruft sich in seiner Überlieferung auf einen Begleiter Alexanders, Aristobulos von Kassandreia. In dessen Alexander-Vita soll dieser den Knoten schlau dadurch gelöst haben, dass er den Deichselnagel herauszog und damit das Joch wegziehen konnte.

Der Bezug zum Gesundheitswesen? Hier fallen die Analogien zum Gordischen Knoten angesichts der verschiedensten System-Paradoxien als Folge von Steuerungspluralität und Interessensvielfalt nicht schwer. Bisweilen entsteht der Eindruck, dass die verknoteten Interessensphären von den Akteuren nicht aufgelöst, sondern von Mal zu Mal fester angezogen werden. Auf der einen Seite ziehen die Leistungserbringer, mit ihrer sektorenspezifischen Rationalität bisweilen in unterschiedliche Richtungen, auf der anderen Seite verfolgen die Financiers der gesetzlichen und privaten Krankenversicherungen ihre Ziele, die oft genug auch von Teilzielen wie der Beitragssatzstabilität und gesamtwirtschaftlichen Interessen geprägt sind. Und haben nicht auch die Bürgerinnen und Bürger vielfältige Rollen mit wechselnden Interessenslagen, mal als (noch) gesunde Versicherte, mal als Patienten, mal als Angehörige [1]? Auch die ordnende Hand der gesundheitspolitischen Mandatsträger ist politischen Kalkülen bzw. kurzfristigen Zielvorgaben ausgesetzt und hat damit bisweilen nur geringen Spielraum.

Mit welcher Kunstfertigkeit wurde der Knoten zwischen den Sozialpartnern eigentlich ursprünglich geflochten? Der geniale Wurf des Bismarckschen Sozialversicherungssystems lässt sich deutlich an der gesetzlichen Unfallversicherung von 1884 erkennen. Hier hat der Staat für sich selbst ausgabenneutral verfügt, dass eine Unfall-Pflichtversicherung der Arbeitsgeber für Ihre Arbeitnehmer zu finanzieren sei und damit die - für Arbeitnehmer oft schwer einzufordernde - Unternehmerhaftpflicht abgelöst. Hintergrund waren die häufigen, in ihrer sozialen Kon- sequenz oftmals weitreichenden Folgen von Arbeitsunfällen in der frühen Phase der Industrialisierung. Die Folgen für die Familien führten nicht zuletzt zu den sozialen Unruhen, welche der kaiserlichen Botschaft von 1881 vorangegangen waren. Die finanzielle Verantwortung der Arbeitgeber bei Arbeitsunfällen sowohl für die Behandlungs- und Rehabilitationskosten als auch für die sozialen Ausgleichszahlungen, beförderte deren Eigeninteresse an einer Reduktion der Unfallzahlen und führte zusammen mit dem gewerkschaftlichen Kampf und den sozialpolitischen Reformen nach dem ersten Weltkrieg zu einer tiefgreifenden Humanisierung der Arbeitswelt. Bis heute sind die Berufsgenossenschaften und Unfallkassen in diesem Feld vorbildlich tätig. Der Unfallversicherungsbeitrag der Arbeitgeber kann somit, abgestuft nach Risiken, innerhalb dieser Interessensgemeinschaft niedrig gehalten werden, eine win-win-Situation. In der Folge sind heute Haus und Garten oft weniger sicher als die von den Unfallversicherungsträgern und Arbeitsschutzbehörden überwachten Arbeitsplätze.

Dies lässt sich teilweise auch der Zunahme an White-Collar-Arbeiten an Bildschirmen und Bürotischen zuschreiben. Doch ergibt sich hieraus nicht eine neue Verantwortung für die Arbeitgeber, welche sich im Vergleich zu früher nun mit chronischen Krankheiten wie Diabetes mellitus und deren Risikofaktoren wie Bewegungsmangel konfrontiert sehen? Oder mit so mancher aus der privaten Lebenssphäre wie aus der Arbeitsbelastung heraus entstandenen Beeinträchtigung der psychischen Gesundheit? Die Ansätze der betrieblichen Gesundheitsförderung gehen hier einen richtigen präventiven Schritt. Positive Erfahrungen im Umfeld der betrieblichen Gesundheits- und Präventionskultur haben u.a. auch zur Propagierung des Setting-Ansatzes in Kitas, Schulen, Universitäten, Stadtvierteln und anderen Lebenswelten geführt.

Um auch für diese neuen Settings win-win-Situationen herzustellen: Wäre für die Verhältnisprävention im öffentlichen Raum nicht eine Steuerfinanzierung z.B. über eine Stärkung des öffentlichen Gesundheitsdienstes eine mögliche Variante? Wäre bei individueller Verhaltensprävention nicht auch unter Anerkennung der Eigenverantwortung eine Prämienrückerstattung mit sozialem Augenmaß denkbar? Könnte nicht eine Besteuerung von gesundheitsschädlichem, übertriebenem Zucker-, Salz- und Fettgehalt die externalisierten Gesundheitskosten zumindest teilweise wieder auf den Produzenten zurückführen? „Was bringt den Doktor um sein Brot?/ a) 
die Gesundheit, b) der Tod/Drum hält der Arzt, auf dass er lebe/uns zwischen beiden in der Schwebe! " (Eugen Roth). Sind auf Seiten der Leistungserbringer in der ambulanten, stationären, pflegerischen und rehabilitativen Versorgung die Anreize wirklich schon optimal gesetzt? Haben „disruptive“ Technologien, welche ein „mehr“ an Gesundheit durch ein „weniger“ an Ressourcenaufwand erreichen können, in einem marktorientierten Gesundheitswesen eine Chance [2]? Der Ökonom Kenneth Boulding hat auf eine Unterscheidung zwischen a) der ökonomischen Sphäre einer marktmäßigen Tauschinteraktion im klassischen ökonomischen Sinn von b) einer Ökonomie, die auf Drohung und Abschreckung basiert, sowie von c) einer Ökonomie im Zusammenhang mit Liebe und Fürsorge hingewiesen [3]. Weite Bereiche des Gesundheitswesens gehören letzterer Sphäre an - „mehr Markt macht [eben] nicht gesund" [4,5]. Wobei im genossenschaftlichen Gesundheitsmarkt bundesdeutscher Prägung an die Stelle einer abstrakten „unsichtbaren Hand“ bisweilen ein konkreter unsichtbarer Handschlag zwischen den Akteuren getreten zu sein scheint und diesbezüglich transparente Strukturen der Kooperation wie auch der Konfliktbewältigung, ggf. auch strafrechtliche Einhegungen, zu fordern sind.

Hat unser Gesundheitswesen eine Komplexität erreicht, in welcher unser analytisches Denken an Grenzen stößt? Welche Steuerung ist angesichts eines solch verwickelten gordischen Knotens aus in Teilen auch berechtigten Eigeninteressen auf unterschiedlichen Ebenen noch möglich? Kommen nicht durch Innovationen aus Wissenschaft und Forschung ständig neue Herausforderungen auf unser Gesundheitswesen zu? Zum Beispiel der Wunsch nach einer sprechenden Medizin nicht nur in $\mathrm{Zu}-$ sammenhang mit den Erkenntnissen der Psychoneuroimmunologie, sondern auch im Interesse einer verbesserten Diagnostik; die Hoffnungen einer personalisierten Medizin in Zusammenhang mit Genomik und Epi-Genetik; darüber hinaus der Ruf nach einer präventiven Wende im Gesundheitswesen, um mithilfe der Public Health-Bezugswissenschaften „Bedingungen zu schaffen, in denen Menschen gesund sein können“ (Institute of Medicine)?

Vielleicht könnte eine Orientierung an konsensualen Systemzielen für ein faires Zusammenspiel im „System Gesundheitswesen“ (Alber) helfen. Vorschläge dafür? An erster Stelle das Systemziel eines „mehr“ an Gesundheit, und das für alle. Diese übergeordnete Zielsetzung verfolgt eine Eingrenzung (mikro-)ökonomischer Teil-Zielsetzungen und betont dem gegenüber gesundheitlichen Nutzen, Solidarität und gesellschaftliche Fairness [5]. Ein zweites naheliegendes Ziel ist Nachhaltigkeit mit ihren wirtschaftlichen, politischen und sozialen Facetten. Hierunter fallen eine ökonomisch sinnvolle, sozial gerechte und in der Bevölkerung akzeptierte und damit nachhaltige Finanzierungssystematik, Ressourcenallokation, Produktion und Verteilung von Gesundheitsgütern als Anspruch an ein langlebiges, adaptives und auf die Bedürfnisse der Beteiligten eingehendes System. Ein drittes großes Systemziel ist die Wahrung und Weiterentwicklung der Entscheidungs- und Gestaltungsfreiheit. Diese drückt sich auf Patientenseite in Elementen wie freier Arzt-, Krankenhaus- und Versicherungswahl, informierter Autonomie bei Behandlungsentscheidungen, der Bejahung der Eigenverantwortung und dem Respekt vor der Menschenwürde aus. Darunter fällt auch die Bewahrung der Heilberufe als grundsätzlich freie Berufe. Weiter zu fördernde Aspekte sind ein freier Austausch von Information und eine freie Kommunikation einschließlich eines auch anwaltschaftlichen Eintretens für Randgruppen und Belange der öffentlichen Gesundheit, die Einforderung von wissenschaftlicher Evidenz, Transparenz und Rechenschaftspflichtigkeit sowie Mechanismen der Konfliktbewältigung. (Mehr) Gesundheit für alle, Nachhaltigkeit, Entscheidungs- und Gestaltungsfreiheit - wichtige Systemziele unserer Sozialversicherung Bismarckscher Prägung (siehe auch [6]).

Solchen Steuerungsfragen, den damit verbundenen Auswirkungen und den zu schaffenden Voraussetzungen gehen die Artikel dieser Ausgabe wieder nach: mit Beiträgen zur nachhaltigen Implementierung evidenzbasierter Programme in der Gesundheitsförderung, zur Inanspruchnahme von Früherkennungsuntersuchungen bei Kindern in Sachsen-Anhalt, zur Akzeptanz standardisierter Patientenschulungen am Beispiel der Rückenschule, zur Qualitätssicherung in der sozialmedizinischen Begutachtung, zur betrieblichen Gesundheitsförderung in Unternehmen des Gesundheitssektors, zur betrieblichen Gefährdungsbeurteilung psychischer Belastungen, zu den Kosten ambulant-sensitiver Krankenhausfälle sowie gleichsam forschungsintern zur neuen TIDieR-Checkliste für eine verbesserte Interventionsbeschreibung in Studien.

Um noch einmal auf den Gordischen Knoten zurückzukommen: Auch wenn Alexander mit dem Schwert seinen siegreichen Asienfeldzug markant eröffnete - wir sollten uns bei den komplexen Sachzusammenhängen im Gesundheitswesen vor allzu schnellen Lösungen hüten. Die Weiterentwicklung im Gesundheitswesen gleicht eher der Evolution im Systemwettbewerb und dem berühmten „starken langsamen Bohren von harten Brettern mit Leidenschaft und Augenmaß zugleich" als einer Revolution mit dem Schwert [7]. Allerdings ermöglichen erst die notwendigen Rahmenbedingungen von Ausstiegs-, Artikulationsund Wahlmöglichkeit (exit, voice, choice/loyalty [8]) ein Umsatteln auf erfolgreichere Modelle im Dienste an der Gesundheit für alle. Hierfür ist die Analogie eher die Erzählvariante des „Herauslösens des Deichselnagels“ gegenüber der allgemeinen Wohlfahrt abträglichen Prozessen und Strukturen und das systemische Einfädeln von erfolgversprechenderen Angeboten. Das noch treffendere Bild wäre allerdings das „Umstricken“ des Knotens bei laufender Fahrt: Schließlich muss der Wagen der gesundheitlichen Versorgung ja weiter gezogen werden.

\section{Literatur}

1 Wildner M, den Exter AP, van der Kraan WGM. The changing role of the individual in social health insurance systems. In: Saltman RB, Busse R, Figueras J, (Hrsg.). Social health insurance systems in western Europe. Maidenhead: Open University Press; 2004

2 Bower JL, Christensen CM. Disruptive Technologies: Catching the Wave. Harvard Business Review 1995; 69: 19-45

3 Boulding KE. A preface to grants economics. The economy of love and fear. Praeger Scientific; New York: 1981

4 Hengsbach F. „Mehr Markt” macht nicht gesund - Gesellschaftliche Risiken und solidarische Sicherung entsprechen einander. Gesundheitswesen 2008; 70: 339-359

5 Kuhn J. Wenn sich Therapie nicht lohnt: Gesundheit als ökonomisches Optimierungsproblem? Forum Kritische Psychologie 2009; 53: 50-54

6 Zacher HF. Der deutsche Sozialstaat am Ende des Jahrhunderts. In: Leibfried S, Waagschal U, (Hrsg.). Der Deutsche Sozialstaat - Bilanzen, Reformen, Perspektiven. Frankfurt am Main: Campus Verlag; 2000: S. 53-S. 90

7 Weber M. Politik als Beruf. Reclam; Stuttgart: 1992

8 Hirschman AO. Exit, Voice, and Loyalty: Responses to Decline in Firms, Organizations, and States. Cambridge, MA: Harvard University Press; 1970 